\title{
AHP MODIFICATION FOR DECISION MAKING UNDER UNCERTAINTY
}

\author{
Alexander V. Bochkov \\ LLC NIIgazeconomika, \\ Moscow, Russia \\ E-mail: a.bochkov@gmail.com \\ Nikolay N. Zhigirev \\ LLC NIIgazeconomika, \\ Moscow, Russia \\ E-mail: nnzhigirev@mail.ru
}

\begin{abstract}
The AHP-method proved its efficiency in different situations of decision making under conditions when experts can perform pairwise comparisons of compared objects under study. But, our real life consists of uncertainties. How can we compare the objects if information about them is not available or extremely uncertain? Pairwise comparisons are impossible. In this case, we propose to use the approach of minimizing the functional «errors» in the evaluation of comparable objects (F-ratio test). Through successive iterations initially given equal weight values compared objects are close to the true value (within the stated error). This method does not require the consistency of the pairwise comparisons matrix and, moreover, can be applied to AHP incomplete matrix. The iterations are repeated as long as the new changes do not result in improved objects estimates. The practical example of the method use is considered.
\end{abstract}

Keywords: AHP, objects estimates, uncertainty, F-ratio test, incomplete matrix 
ISAHP Article: Bochkov, Zhigirev/AHP Modification for Decision Making Under Uncertainty/ International Symposium of the Analytic Hierarchy Process 2014, Washington D.C., U.S.A.

\section{Introduction}

Huge number of tasks in different fields of knowledge (such as: definition of relative weight purposes during carrying out multicriteria optimization; definition of expected result of activity, for example determination of investment project efficiency; definition of probabilities of implementation of accident development various scenarios in potentially dangerous object in the probabilistic analysis of its safety; a task of probabilities of outcomes in matrix of consequences at a choice of rational decision in the conditions of partial uncertainty, etc.) is solved in conditions when the behavior of studied process branches and it is necessary to define weights (relative probabilities) of possible scenarios (compared objects). In other tasks some parameters of mathematical models can accept various values depending on some not formalized (not described mathematically) factors. Definition of probabilistic distributions of these parameters is often impossible due to the lack of rather representative statistics. For value assignment of these parameters qualitative or qualitative and quantitative scales are used.

In all considered cases (both a scenario choice, and class definition or object state in some scale) mathematically the task is reduced to compared objects choice from a set possible by means of expert estimation. Whereby the interest is not only in choosing of the most probable compared objects, but also in defining compared objects weight (relative probabilities). This information is used further in algorithms of simulation modeling, and in simpler models in averaging operations taking into account compared objects weight.

Simplicity of relative compared objects weight calculation at their determination accuracy preservation often plays considerable part. The analysis shows that the most reliable and widely used method of problem solution of choice and definition of relative compared objects weight (relative probabilities) is the pairwise comparisons method on qualitative attribute with quantitative assessment of preferences [1].

\section{Literature Review}

The pairwise comparisons method [1] had broad application when determining relative indicators of compared objects importance against chosen qualitative criterion in decision-making support systems. Concerning some general property for compared objects [2-5] this method allows to carry out their ranging, to define a priority of one compared object (criterion, purpose, alternative) before another. One of the most developed and widely practiced is pairwise comparisons method modification, received the name of T. Saati's Analitic Hierarchy Process (AHP) [6].

One of essential shortcomings of pairwise comparisons method various realization is the essential increase in labor input of necessary calculations, with growth of number of estimated objects. In some cases it is too difficult for an expert to perform a large number of pairwise comparisons or he can't simply compare two objects on the offered preferences scale. 
ISAHP Article: Bochkov, Zhigirev/AHP Modification for Decision Making Under Uncertainty/ International Symposium of the Analytic Hierarchy Process 2014, Washington D.C., U.S.A.

There is a problem of processing not completely certain pairwise comparisons matrixes. The solution of this task is connected to the following problems [7]. The first problem is in definition of necessary and sufficient conditions of calculation of all components of compared object importance indicators vector, and the second one is in definition of the algorithm of these components calculation itself.

In [8] these problems are solved concerning of pairwise comparisons results processing in fundamental scale by Eigenvector Following method. The method offered in [8], in point of fact, is reduced to restoration of full matrix of comparisons on available incomplete matrix and application standard algorithm of relative compared objects weight to the restored matrix. For method justification it is offered to use graph interpretation of pairwise comparisons method. Tops of graph $G$ designate alternatives $A_{i} \in A, i=\overline{1, k}$ from which $\operatorname{arcs} d_{i j}$ release, connected them with each other. It is proved that $d_{i j}$ is equal to geometrical average intensity of all possible ways connecting tops $A_{i}$ and $A_{j}$. Necessary and sufficient condition for this method realization: between any pair of tops in graph $G$ there has to be at least one way.

\section{Problem Statement}

We consider the problem of compared objects weight definition importance according to incomplete pairwise comparisons matrixes. In practice most precisely pairwise comparisons are given by experts between "the most significant objects and other objects" and between "the least significant objects and others", that is when differences between pairwise compared objects are contrast. When compared objects are close "naturally" to comparisons matrix element the expert, according to the scale [6], assigns value 1 that leads, in our opinion, to unjustified alignment of compared objects weight estimates.

We offer to consider, in this case, pairwise comparisons uncertain. Whereby instead of procedure of recovery of such uncertain data we offer reverse procedure lying in the fact that to find such subset of pairwise comparisons which is most coordinated and this subset, finally, assign values of compared objects weight. Thereby two aims are achieved. First, the required decision in the form of distribution of compared objects weight importance will be with some set accuracy is coordinated with pairwise comparisons matrix constructed on AHP method. Secondly, the remained pairwise comparisons will indicate communication circuits between compared objects, explaining distinctions of defined weights

So, the task is set as follows: not completely filled pairwise comparisons matrix is set and some initial (for example, uniform) distribution of weights importance objects $W_{i}$ is set . The integrated majorant criterion of these weights mismatch and available at every moment submatrix of comparisons matrix is formulated $-P$.

It is necessary to construct iterative algorithm of ejection of the smallest number of least coordinated pairwise comparisons with simultaneous correction of objects weights $W_{i}$ so 
ISAHP Article: Bochkov, Zhigirev/AHP Modification for Decision Making Under Uncertainty/ International Symposium of the Analytic Hierarchy Process 2014, Washington D.C., U.S.A.

that mismatch criterion $P$ decreases to some acceptable level (probably equal to zero). Final distribution of compared objects weights, received as a result of such adaptive iterative procedure has to be considered as the required solution.

\section{Methodology Description}

Before describing offered algorithm, we will give some important definitions.

Definition 1. $N$-dimension of problem, amount of compared objects $O_{i}=\left\{O_{1}, \ldots, O_{N}\right\}$.

Definition 2. $V=\left\{v_{1}, \ldots, v_{N}\right\}-$ set of tops of estimated compared objects communication graph.

Definition 3. $W_{i}=W\left(V_{i}\right)>0$ - normalized positive estimate of importance $i$ object (compared objects).

Normalization condition:

$$
\sum_{i} W_{i}=1
$$

Definition 4. $H=H(V, V)-N \times N$ dimencionality communication graph.

Whereby $H_{i j}=1$, if $V_{i}$ and $V_{j}$ are connected, $H_{i j}=0$, if such connection lacks due to lack of pairwise comparisons of $O_{i}$ and $O_{j}$. It is estimated that communication graph $H$ is related graph.

Definition 5. $G=G(V, V)$ - subgraph, is named spanning tree of graph $H$, if there is only one way from any top $V_{k}$ to any top $V_{l}$.

Naturally quantity of spanning tree edges (quantity of pairwise comparisons) in spanning tree is equal to $N-1$. In general spanning tree is given by "snowflake" (Fig. 1).

Definition 6. To every nonzero communication graph edge $G$ is given estimate $S_{i j}$, showing pairwise comparisons

$$
W_{i}=S_{i j} \cdot W_{j} .
$$

Please note if spanning tree is chosen then $N-1$ equality (2) and normalization requirement (1) uniquely allow to calculate all $W_{i}$. According to found $W_{i}$ estimations $\widetilde{S}_{i j}$ are defined for other communication graph edges not including into spanning tree. The problem is that these estimations are not the same as pairwise comparisons estimations $S_{i j}$, given by experts. 


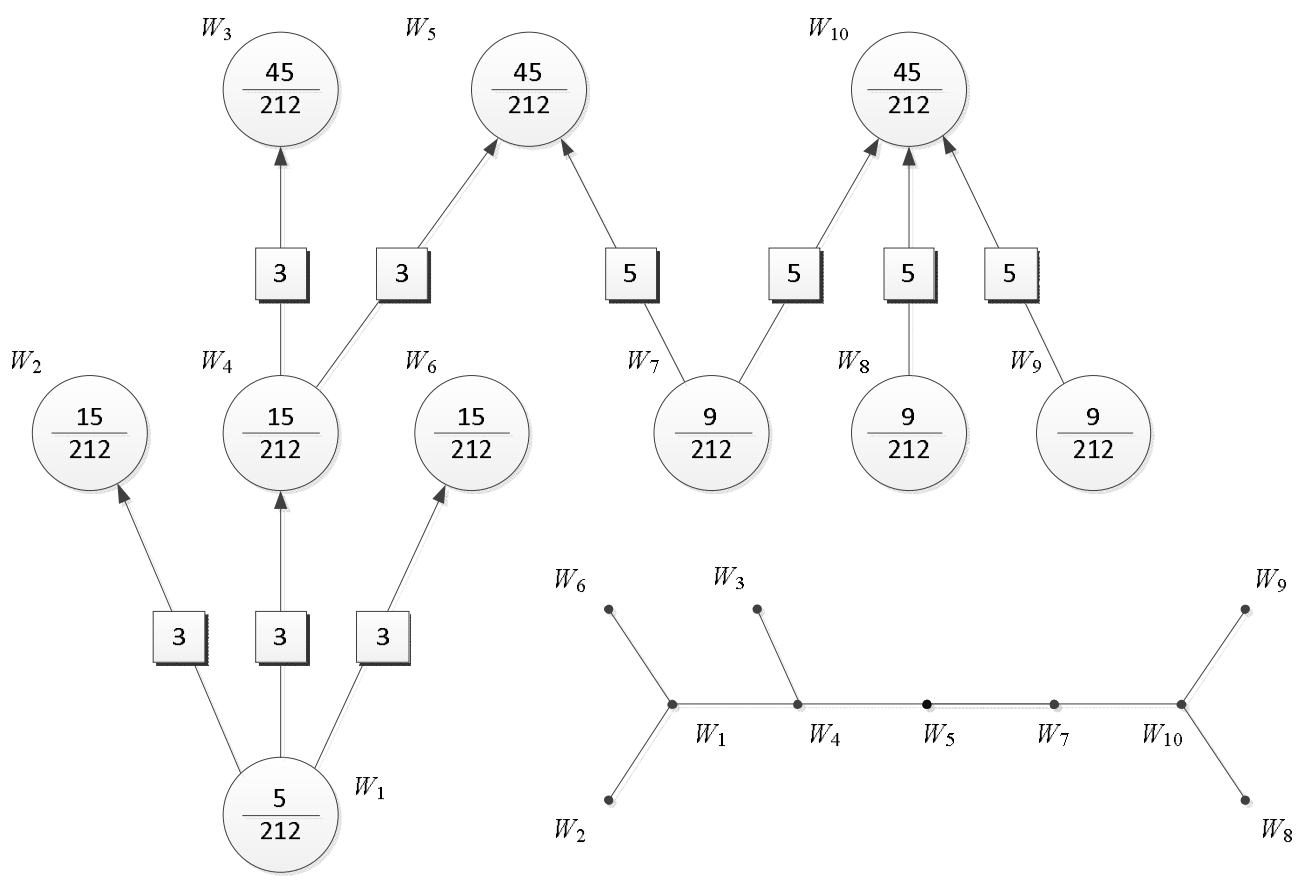

Fig. 1. Spanning tree (in circles - calculated weights of objects importance (tops), in squares - coefficients of pairwise comparisons of conditional example)

If connectivity graph is fully connected graph with $N$ tops, relevant to Saati's method then quantity of spanning tree is estimated by Cayley formula with value $N^{N-2}$ [9]. Thereby search for optimal spanning tree in some multitude similarity metrics $\left\{\widetilde{S}_{i j}\right\}$ and $\left\{S_{i j}\right\}$ by enumeration presents certain technical problems.

Simultaneously it must be admitted that pairwise comparisons estimations given by experts are rather crude and for high dimensions $N$ «mechanistic». Thereby it is incorrectly to require proximate satisfaction of equalities (2) for all $H$ graph edges, and in this way for edges entered into spanning tree $G$.

In this context, we take compelled step - we weaken conditions (2), allowing for tops $V_{i}$ and $V_{j}$ through $S_{i j}$ mensurate opponent with some default level. Herewith of course it needs to consider that similarly measuring is asymmetric, i.e. the fact is not so critical that the object with big importance gets through another less important object importance estimation which is higher as it is. Also it is not critical when the object with little importance is underestimated because of more important objects.

Thus, for every communication graph edge we shall estimate value 
ISAHP Article: Bochkov, Zhigirev/AHP Modification for Decision Making Under Uncertainty/ International Symposium of the Analytic Hierarchy Process 2014, Washington D.C., U.S.A.

$$
f_{i j}=\frac{W_{i}}{S_{i j} \cdot W_{j}}-1
$$

"Ideally" all $f_{i j}$ must be equal to zero, as for us we require them to be in some window with width $2 P$. Here $P$ is important parameter of suggested method which while solution construct shall go to zero (note that zero is realizable if we take decision on solution determination specifically in the shape of spanning tree). In addition, not to lose intergrating characteristics of pairwise comparisons, it is sufficient to concentrate on some level $P_{f}$, different from zero a little. In this situation connected subgragh $H(p)$ of graph $H$ will be formed, exhibiting the characteristic when all estimations $f_{i j}$ are set in window with width $2 P_{j}$.

Take into account skewness of estimating $f_{i j}$ for different values we consider two intervals $R\left(W_{i}\right)$ and $L\left(W_{i}\right)$ add up to size $P$ (Fig. 2):

$$
L\left(W_{i}\right)+R\left(W_{i}\right)=2 P
$$

Fig.2.

We will consider that for big $W_{i}$ values the centre of window displaces to the right of zero and for little values $W_{i}$, respectively - to the left of zero. Herewith in "middle" position $\left(W_{i}=\frac{1}{N}\right)$ displacement is not occurred. Value of right border $R\left(W_{i}\right)$ under $W_{i}=1$ we'll consider equal to $1,9 P$, left one, respectively $-0,1 P$. To the contrary for little $W_{i}$ values right border is "forced against" level $0,1 P$, and left border stands off zero at a distance of $1,9 P$.

We suggest plotting correspondence in the shape of hyperbola:

$$
R\left(W_{i}\right)=0,1 P+1,8 P \cdot \frac{(N-1) \cdot W_{i}}{1+(N-2) \cdot W_{i}} .
$$

At first for big values $P$ inequations

$$
-L\left(W_{i}\right) \leq f_{i j} \leq R\left(W_{i}\right)
$$

are performed for all communication graph edgs.

International Symposium of

6

Washington, D. C. the Analytic Hierarchy June 29 - July 2, 2014 
ISAHP Article: Bochkov, Zhigirev/AHP Modification for Decision Making Under Uncertainty/ International Symposium of the Analytic Hierarchy Process 2014, Washington D.C., U.S.A.

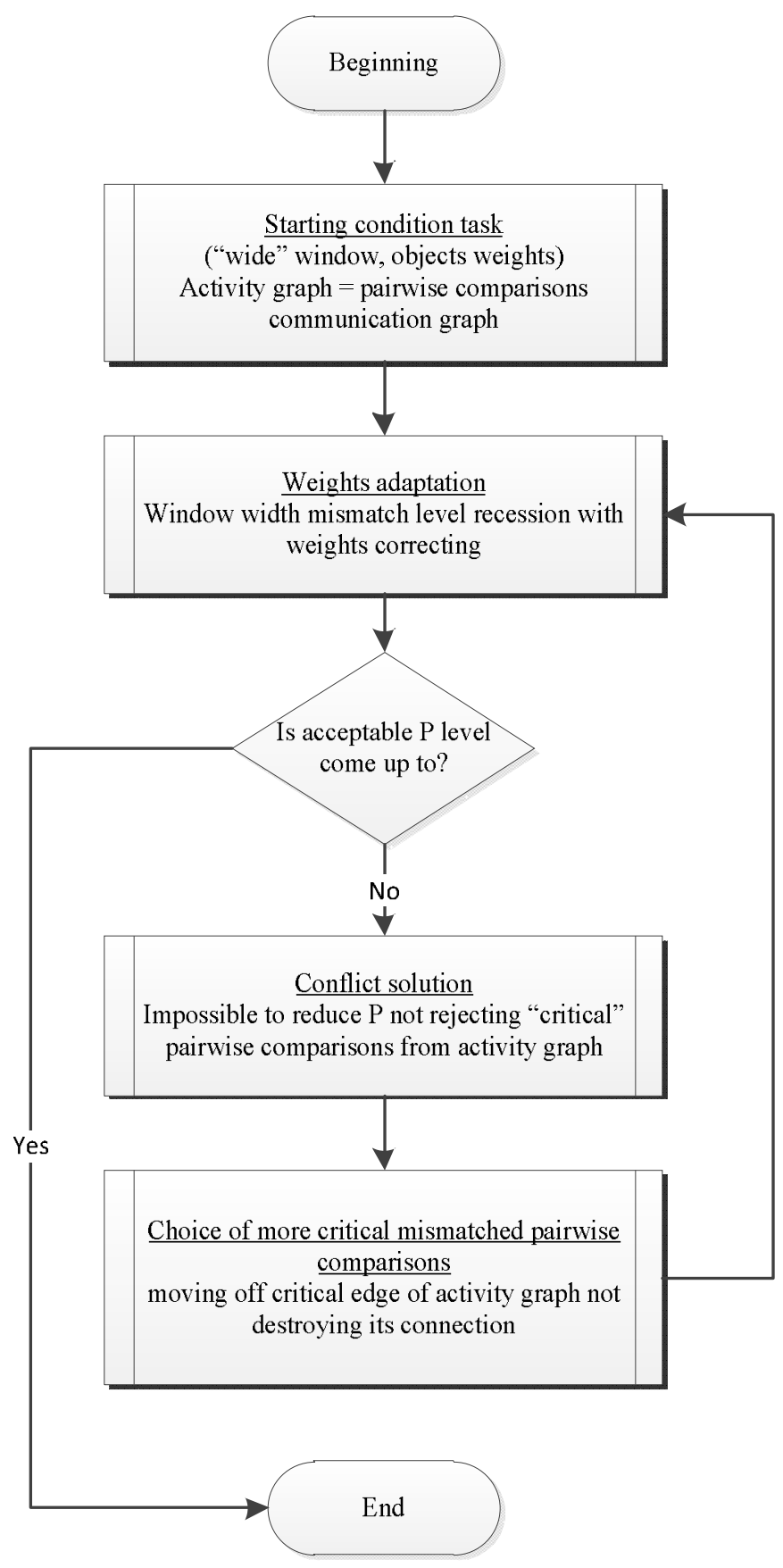

Fig. 3. Bookkeeping scheme algorithm 
ISAHP Article: Bochkov, Zhigirev/AHP Modification for Decision Making Under Uncertainty/ International Symposium of the Analytic Hierarchy Process 2014, Washington D.C., U.S.A.

While reduction $P$ exit vertex of one or several estimations $f_{i j}$ will occur to the borders of windows. While $f_{i j}$ exit to the right border of the window $R\left(W_{i}\right)$ value $W_{i}$ must be extended, herewith the centre of this window will move to the right as simultaneous reduction of all other weights. While $f_{i j}$ exit to the left border, $W_{i}$ must be reduced with simultaneous increase of weights of all other tops.

Adaptation of tops weights in $G$ coupling matrix happens. At the cost of this adaptation further reduction of $P$ value contributes, which as the final result ends in situation when all tops $W_{i}$ have even one edge situated according to its estimation $f_{i j}$ at the border of relevant window. Correlation conflict happens. It is impossible to move even one edge $G_{i j}$ inside window not "pushing out" any other edge $G_{k l}$ in the amount of reached default level $P$. In this situation one of critical edges must be moved off coupling matrix. It is clear this edge mustn't be spanning, i.e. $G$ graph connectivity mustn't be lost. By experiment we move off such edge $G_{i j}$, for which another edge $G_{i k}$ exists very close to the border of the window. As a result all $f_{i j}$ adjoined to top $V_{i}$ constitute content to relevant border of the window, and make it possible to continue adaptation process, changing both value $W_{i}$ and value $W_{j}$, by virtue of the fact that $V_{j}$ after critical coupling release also became "less bounded".

Omitting detailed descriptions in this article we give bookkeeping scheme algorithm general view of method under discussion (Fig. 3).

\section{Application example}

We will consider control-flow chart operation by the example of pairwise comparisons matrix processing of ten objects. Basic data are given in Tab.1.

From 45 pairwise comparisons 18 comparisons are passed (NA). Other 27 estimations indicate preference of objects $\mathrm{O}_{3}$ and $\mathrm{O}_{5}$ over other objects, but mismatch of these estimations demanded clarification of the following circumstance: whether these estimations are enough to exclude demonstratively from leaders, let us say, object $\mathrm{O}_{10}$.

We will note that the example is taken from authors' real task decision practice, instead of specially prepared for illustration of offered method opportunities.

So, initially choosing weights of all objects equal 0,1 through an exception of edges with simultaneous reduction of the size of the window $P$, the decision $W(O)$ provided in Fig. 1 and in two last columns of Tab. 2 (in the form of standard fraction and in a decimal form) is constructed. 
ISAHP Article: Bochkov, Zhigirev/AHP Modification for Decision Making Under Uncertainty/ International Symposium of the Analytic Hierarchy Process 2014, Washington D.C., U.S.A.

Table 1

\begin{tabular}{|c|c|c|c|c|c|c|c|c|c|c|}
\hline & $\mathrm{O}_{1}$ & $\mathrm{O}_{2}$ & $\mathrm{O}_{3}$ & $\mathrm{O}_{4}$ & $\mathrm{O}_{5}$ & $\mathrm{O}_{6}$ & $\mathrm{O}_{7}$ & $\mathrm{O}_{8}$ & $\mathrm{O}_{9}$ & $\mathrm{O}_{10}$ \\
\hline $\mathrm{O}_{1}$ & & $\frac{1}{3}$ & $\frac{1}{5}$ & $\frac{1}{3}$ & $\frac{1}{7}$ & $\frac{1}{3}$ & $\mathrm{NA}$ & $\mathrm{NA}$ & $\mathrm{NA}$ & $\frac{1}{4}$ \\
\hline $\mathrm{O}_{2}$ & $\frac{3}{1}$ & & $\frac{1}{5}$ & $\mathrm{NA}$ & $\frac{1}{5}$ & $\frac{1}{4}$ & $\mathrm{NA}$ & $\mathrm{NA}$ & $\mathrm{NA}$ & $\frac{1}{3}$ \\
\hline $\mathrm{O}_{3}$ & $\frac{5}{1}$ & $\frac{5}{1}$ & & $\frac{3}{1}$ & $\mathrm{NA}$ & $\frac{3}{1}$ & $\frac{3}{1}$ & $\frac{5}{1}$ & $\frac{5}{1}$ & $\frac{3}{1}$ \\
\hline $\mathrm{O}_{4}$ & $\frac{3}{1}$ & $\mathrm{NA}$ & $\frac{1}{3}$ & & $\frac{1}{3}$ & $\mathrm{NA}$ & $\mathrm{NA}$ & $\mathrm{NA}$ & $\mathrm{NA}$ & $\frac{1}{3}$ \\
\hline $\mathrm{O}_{5}$ & $\frac{7}{1}$ & $\frac{5}{1}$ & $\mathrm{NA}$ & $\frac{3}{1}$ & & $\frac{3}{1}$ & $\frac{5}{1}$ & $\frac{5}{1}$ & $\frac{5}{1}$ & $\frac{3}{1}$ \\
\hline $\mathrm{O}_{6}$ & $\frac{3}{1}$ & $\frac{4}{1}$ & $\frac{1}{3}$ & $\mathrm{NA}$ & $\frac{1}{3}$ & & $\mathrm{NA}$ & $\mathrm{NA}$ & $\mathrm{NA}$ & $\frac{1}{4}$ \\
\hline $\mathrm{O}_{7}$ & $\mathrm{NA}$ & $\mathrm{NA}$ & $\frac{1}{3}$ & $\mathrm{NA}$ & 0,2 & $\mathrm{NA}$ & & $\mathrm{NA}$ & $\mathrm{NA}$ & $\frac{1}{5}$ \\
\hline $\mathrm{O}_{8}$ & $\mathrm{NA}$ & $\mathrm{NA}$ & $\frac{1}{5}$ & $\mathrm{NA}$ & $\frac{1}{5}$ & $\mathrm{NA}$ & $\mathrm{NA}$ & & $\mathrm{NA}$ & $\frac{1}{5}$ \\
\hline $\mathrm{O}_{9}$ & $\mathrm{NA}$ & $\mathrm{NA}$ & $\frac{1}{5}$ & $\mathrm{NA}$ & $\frac{1}{5}$ & $\mathrm{NA}$ & $\mathrm{NA}$ & $\mathrm{NA}$ & & $\frac{1}{5}$ \\
\hline $\mathrm{O}_{10}$ & $\frac{4}{1}$ & $\frac{3}{1}$ & $\frac{1}{3}$ & $\frac{3}{1}$ & $\frac{1}{3}$ & $\frac{4}{1}$ & $\frac{5}{1}$ & $\frac{5}{1}$ & $\frac{5}{1}$ & \\
\hline
\end{tabular}

In Tab. 2 you can find the summary data illustrating that estimations mismatch on different steps of algorithm differently influences compared objects importance weights ratios. So, in process of critical edges removal defining these edges estimations mismatch with all set of pairwise comparisons which have remained in working subgraph, these weights values execute a periodic motion relating to final solution given in the last column.

It should be noted that coordination of estimates of objects at $\mathrm{P}=0,742$ level (the second column of Tab. 2) and at $\mathrm{P}=0,469$ level (the third column of Tab. 2) demanded removal of mismatched edges, however begin with $\mathrm{P}=0,207$ level all mismatches were found eliminated and further weights corrections were carried out only at the cost of "revaluation of objects importance weights" adaptation mechanism.

It is also important to note that the first mismatches conflict occurred at $\mathrm{P}=1,15$ level. For overcoming of this conflict communication estimation $\mathrm{O}_{3}-\mathrm{O}_{10}$ was removed. Thus succeedent number of removals occurs as if the algorithm in advance knows the solution constructed on spanning tree edges.

To $\mathrm{P}=0,742, \mathrm{P}=0,469$ and $\mathrm{P}=0,207$ levels given in Tab. 2 visual coloring of Tab. 3 corresponds, in which different shading show measures of disagreement of pairwise estimations without considering correcting displacements $-f_{i j}$ value, mentioned in hypotheses that objects importance weights are the weights found at $P=P_{f}$. 
ISAHP Article: Bochkov, Zhigirev/AHP Modification for Decision Making Under Uncertainty/ International Symposium of the Analytic Hierarchy Process 2014, Washington D.C., U.S.A.

Table 2

\begin{tabular}{|c|c|c|c|c|c|c|c|cc|}
\hline & \multicolumn{7}{|c|}{ Window size } \\
\hline Beca & $\mathrm{P}=0,742$ & $\mathrm{P}=0,469$ & $\mathrm{P}=0,207$ & $\mathrm{P}=0,1$ & $\mathrm{P}=0,05$ & $\mathrm{P}=0,01$ & $\mathrm{P}=0,0001$ & \multicolumn{2}{c|}{$\mathrm{P}=0$} \\
\hline $\mathrm{W}_{1}$ & 0,047 & 0,0447 & 0,026 & 0,026 & 0,025 & 0,0237 & 0,0235 & $\frac{5}{212}$ & $=0,02359$ \\
\hline $\mathrm{W}_{2}$ & 0,084 & 0,0923 & 0,077 & 0,075 & 0,073 & 0,0711 & 0,0707 & $\frac{15}{212}$ & $=0,07075$ \\
\hline $\mathrm{W}_{3}$ & 0,209 & 0,1735 & 0,185 & 0,210 & 0,211 & 0,2110 & 0,2123 & $\frac{45}{212}$ & $=0,21226$ \\
\hline $\mathrm{W}_{4}$ & 0,084 & 0,0923 & 0,077 & 0,075 & 0,073 & 0,0711 & 0,0707 & $\frac{15}{212}$ & $=0,07075$ \\
\hline $\mathrm{W}_{5}$ & 0,209 & 0,1735 & 0,185 & 0,210 & 0,211 & 0,2110 & 0,2123 & $\frac{45}{212}$ & $=0,21226$ \\
\hline $\mathrm{W}_{6}$ & 0,084 & 0,0923 & 0,077 & 0,075 & 0,073 & 0,0711 & 0,0707 & $\frac{15}{212}$ & $=0,07075$ \\
\hline $\mathrm{W}_{7}$ & 0,047 & 0,0357 & 0,045 & 0,042 & 0,042 & 0,0425 & 0,0425 & $\frac{9}{212}$ & $=0,04246$ \\
\hline $\mathrm{W}_{8}$ & 0,031 & 0,0357 & 0,045 & 0,042 & 0,042 & 0,0425 & 0,0425 & $\frac{9}{212}$ & $=0,04246$ \\
\hline $\mathrm{W}_{9}$ & 0,031 & 0,0357 & 0,045 & 0,042 & 0,042 & 0,0425 & 0,0425 & $\frac{9}{212}$ & $=0,04246$ \\
\hline $\mathrm{W}_{10}$ & 0,174 & 0,2243 & 0,238 & 0,203 & 0,208 & 0,2135 & 0,2123 & $\frac{45}{212}$ & $=0,21226$ \\
\hline
\end{tabular}

Table 3

\begin{tabular}{|c|c|c|c|c|c|c|c|c|c|c|}
\hline & $\mathrm{O}_{1}$ & $\mathrm{O}_{2}$ & $\mathrm{O}_{3}$ & $\mathrm{O}_{4}$ & $\mathrm{O}_{5}$ & $\mathrm{O}_{6}$ & $\mathrm{O}_{7}$ & $\mathrm{O}_{8}$ & $\mathrm{O}_{9}$ & $\mathrm{O}_{10}$ \\
\hline $\mathrm{O}_{1}$ & & $\begin{array}{c}\underline{0} \\
1260\end{array}$ & $-\frac{560}{1260}$ & $\begin{array}{c}\underline{0} \\
1260\end{array}$ & VIT" & $\begin{array}{c}\underline{0} \\
1260\end{array}$ & NA & NA & NA & $-\frac{700}{1260}$ \\
\hline $\mathrm{O}_{2}$ & $\begin{array}{c}\underline{0} \\
1260\end{array}$ & & $+\frac{840}{1260}$ & NA & $+\frac{840}{1260}$ & $+\frac{3780}{1260}$ & NA & NA & NA & 3 \\
\hline $\mathrm{O}_{3}$ & $+\frac{1008}{1260}$ & $-\frac{504}{1260}$ & & $\begin{array}{c}\underline{0} \\
1260\end{array}$ & NA & . & $+\frac{840}{1260}$ & 3 & 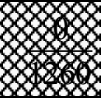 & $-\frac{840}{1260}$ \\
\hline $\mathrm{O}_{4}$ & $\begin{array}{c}\underline{0} \\
1260\end{array}$ & NA & $\begin{array}{c}\underline{0} \\
1260\end{array}$ & & $\begin{array}{c}\underline{0} \\
1260\end{array}$ & NA & NA & NA & NA & m \\
\hline $\mathrm{O}_{5}$ & DI & $-\frac{504}{1260}$ & NA & $\begin{array}{c}\underline{0} \\
1260\end{array}$ & & 3 & $\begin{array}{c}\underline{0} \\
1260\end{array}$ & m & r & $-\frac{840}{1260}$ \\
\hline $\mathrm{O}_{6}$ & $\begin{array}{c}\underline{0} \\
1260\end{array}$ & $\frac{945}{1260}$ & z & NA & 2 & & NA & NA & NA & 1126 \\
\hline $\mathrm{O}_{7}$ & NA & NA & $-\frac{504}{1260}$ & NA & $\begin{array}{c}\underline{0} \\
1260\end{array}$ & NA & & NA & NA & $\begin{array}{c}\underline{0} \\
1260\end{array}$ \\
\hline $\mathrm{O}_{8}$ & NA & NA & m & NA & m & NA & NA & & NA & $\begin{array}{c}\underline{0} \\
1260\end{array}$ \\
\hline $\mathrm{O}_{9}$ & NA & NA & m & NA & m & NA & NA & NA & & $\begin{array}{c}\underline{0} \\
1260\end{array}$ \\
\hline & $-\frac{1575}{1260}$ & m & $+\frac{2520}{1260}$ & m & $+\frac{2520}{1260}$ & Etas & $\begin{array}{c}\underline{0} \\
1260\end{array}$ & $\begin{array}{c}\underline{0} \\
1260 \\
\end{array}$ & $\begin{array}{c}\underline{0} \\
1260\end{array}$ & \\
\hline
\end{tabular}

International Symposium of the Analytic Hierarchy

Washington, D. C. Process June 29 - July 2, 2014 
ISAHP Article: Bochkov, Zhigirev/AHP Modification for Decision Making Under Uncertainty/ International Symposium of the Analytic Hierarchy Process 2014, Washington D.C., U.S.A.

As is obvious in Tab. 2 - beginning with $\mathrm{P}=0,1$ approximate solutions excursions differ from solution according to spanning tree less than for $1 \%$. This circumstance allowed us to display visually $f_{i j}$ sizes, reduced them to a common denominator.

Excluded at the first stage of algorithm work four pairwise comparisons (from $\mathrm{P}=1,15$ to $\mathrm{P}=0,742$ ) are painted in dark grey colour (Tab. 3). Four pairwise comparisons removed at the second stage (from $\mathrm{P}=0,742$ to $\mathrm{P}=0,469$ ) are painted in light grey colour. Two pairwise comparisons removed at the third stage (from $\mathrm{P}=0,469$ to $\mathrm{P}=0,207$ ), are marked with shading. The cells of Tab. 3 containing remained pairwise comparisons are marked with double shading. The cells containing pairwise comparisons, compatible with spanning tree edges, are painted in black colour.

Let us remark that estimations in table cells with double shading coincide with estimations recalculated through pairwise comparisons estimating, compatible with spanning tree edges. Actually existence of such "duplicating" communications in pairwise comparisons matrix as we understand, allows in the course of solution creation through mismatches conflicts elimination to support main preferences framework. Estimations in cells with double shading are rejected at the last stage at $\mathrm{P}$ going to zero for spanning creation.

\section{Results discussion}

Thus, suggested method, without denying AHP, allows to expand its opportunities significantly. At program realization of the algorithm described in the article it is possible to receive express estimations of compared objects importance without completing all pairwise comparisons matrix.

The method allows "to control" AHP matrix completing process as it reveals places of critical experts' estimates mismatch which can be more extensive than mismatch of estimates in threes (as usual among many).

In concept the method allows to work and with uncoordinated matrixes from which they refuse in AHP, in this case the number of iterations of edges exception from graph will naturally increase significantly.

Solution to mismatches conflicts is very similar to Delphi ${ }^{1}$ method: "ponderability estimation dropping out of general series" - that is on the border - we "cancel" and we try to adapt other estimations to each other in "harder" circumstances.

\section{Conclusions}

Thus, we offer the method, allowing to receive compared objects estimations at incomplete comparisons and giving the chance to follow the structure of fundamental comparisons forming objects importance weights. 
ISAHP Article: Bochkov, Zhigirev/AHP Modification for Decision Making Under Uncertainty/ International Symposium of the Analytic Hierarchy Process 2014, Washington D.C., U.S.A.

\section{References}

1. Bradley, R. A. and Terry, M. E. (1952). Rank analysis of incomplete block designs, I. the method of paired comparisons. Biometrika, 39, 324-345.

2. Markin, E.G. (1974). Problem of multiple select. - M.: Nauka (Science), 1974. - p. 256

3. Kendal, M. (1975). Grade correlation. - M.: Statistics, 1975. - p. 216.

4. David, G. (1978). Pairwise comparison method. - M.: Statistics, 1978. - p. 144.

5. Litvak, V. G. (1982). Expert information. Method for obtaining and analysis. - M.: Radio i Svyaz (Radio and communication), 1982. - p. 184.

6. Saati, T. L. (2008). Solution making at correspondences and backlinks: Analytic nets. - M.: Izdatelstvo (Press) LKI, 2008.- p. 360.

7. Totsenko, V. G. (2002). Methods and systems for supporting of solution making. Algorithmic aspect. - Riev: Naukova dumka, 202. - p. 381.

8. Harker, P. T. (1987) Incomplete pairwise comparisons in the analytic hierarchy process, Mathematical Modelling 9 (1987), 837-848.

9. A. Cayley (1889). «A theorem on trees». Quart. J. Math 23:376-378. 